\title{
Total Incremental Method for Solving Nonlinear System of Equation Due to Plasticity of Reissner Plates with Boundary Element Method
}

\author{
Supriyono \\ Departement of Mechanical Engineering, Faculty of Engineering, \\ Muhammadiyah University of Surakarta. \\ Email:supriyono@ums.ac.id
}

\begin{abstract}
In this paper a total incremental method for solving nonlinear system equation due to plasticity of shear deformable plates is presented. The material is assumed to undergo small strains. The von Mises criterion is used to evaluate the plastic zone and elastic perfectly plastic material behaviour is assumed. An initial stress formulation is used to formulate the boundary integral equations. The domain integral due to material nonlinearity is evaluated using a cell discretization technique. Several examples are presented and comparisons are made to demonstrate the validity and the accuracy of the total incremental method to solve the nonlinear system of equation due to plasticity.
\end{abstract}

\section{Key words : Reissner plates-nonlinear system of equation-total increment method-plasticity-boundary element method.}

\section{Introduction}

Nonlinear analysis of plate bending can be divided into two categories e.i. geometrical and material nonlinearity. Geometrical nonlinearity in plate bending usually is called as large deflection.

There are two widely used plate theories. The first one was developed by Kirchhof (1850)] and is commonly referred to as the classical theory. The other was developed by Reissner (1950)], and is known as the shear deformable theory. The classical plate theory neglects the shear deformation through the plate thickness whereas the shear deformable theory takes into account the shear deformation and the transverse normal stresses through the plate thickness. The Reissner theory is based on modelling the plate structure as two-dimensional structure with assumed stress variation through the plate thickness. In Reissner plates, the problem is represented in terms of three degrees of freedom, involving generalized displacements (i.e. two rotations and deflection) and generalized tractions (i.e. moments and transverse shear force).

Nonlinear analysis of plate bending with boundary element method (BEM) can be found in the works by Tanaka (1984), Kamiya and Sawaki (1982), Lei, Huang and Wang (1990), Karam and Telles (1998), Ribeiro and Venturini (1998), Wen, Aliabadi and Young (2004), Dirgantara and Aliabadi (2006), and Purbolaksono and Aliabadi (2005) and Supriyono and Aliabadi (2006).

The works by by Tanaka, Kamiya and Sawaki and Lei, Huang and Wang dealt with large deflection using classical plate theory. Whereas the works by Wen, Aliabadi and Young, Dirgantara and Aliabadi and Purbolaksono and Aliabadi were for large deflection analysis using Reissner plates theory. The BEM analysis of Reissner plates with material nonlinearity can be found in the works by 
Karam and Telles and Ribeiro and Venturini. Supriyono and Aliabadi developed the application of the BEM for Reissner plates by considering combined large deflection and plasticity.

An incremental together with an iterative procedure is usually applied in delaing with nonlinear system of equation. However, Wen, Aliabadi and Young (2004) proposed the total incremental method to solve the nonlinear system of equation due to large deflection in which the iterative process is neglected. Nevertheless, the size of the increment should be small and depends on the problems being analyzed.

Purbolaksono and Aliabadi (2005) studied four methods of solution for the nonlinear problem due to large deflection which included total incremental method, cumulative load incremental method, Euler method and nonlinear system method. They found the most efficient approach is the total incremental method proposed by Wen, Aliabadi and Young (2004) which has much simpler algorithm and less computer time compared to the incremental and iterative method.

The success application of the total incremental method in large deflection analysis suggests that an extension of the method into plasticity analysis my also be effective. This paper presents the application of the total incremental method to solve the nonlinear system of equation due plasticity in BEM. The BEM formulation in this work follows closely the work by Karam and Telles (1998). An initial stress formulation was used and von Mises yield criterion is applied to eavulate plastic zone. The formulation allows for small strain. Elastic perfectly plastic material is considered and cell discretization approach was applied to evaluate the domain integral. However, in this work higher order cell, which is 9-nodes quadrilateral cell, is considred instead of triangular constant cell. Throughout this paper, the cartesian tensor notation is used, with Greek indices varying from 1 to 2 and the Latin indices varying from 1 to 3 .

\section{Displacement and Stress Integral Equations}

Applications BEM in solid mechanics are based on the Somigliana's identities. Somigliana's identity for displacements in elastoplastics shear deformable plate bending problems states that the rate of the displacements (two rotations and one deflection) at any points $X^{\prime}\left[\dot{w}_{i}\left(X^{\prime}\right)\right]$ that belong to domain $\left(X^{a} V\right)$ to the boundary values of displacement rates $\left[\dot{w}_{i}(x)\right]$ and traction rates [ $\dot{p}_{j}(x)$ ] can be expressed as (Karam, 1998):

$$
\begin{aligned}
\dot{w}_{i}(X)= & \int_{S} W_{i j}\left(X^{\prime}, x\right) \dot{p}_{j}(x) d S-\int_{S} P_{i j}\left(X^{\prime}, x\right) \dot{w}_{j}(x) d S+ \\
& \int_{V} W_{i 3}\left(X^{\prime}, X\right) \dot{q}_{3}(X) d V+ \\
& \int_{V} \chi_{\alpha \beta i}\left(X^{\prime}, X\right) \dot{M}_{\alpha \beta}^{p l}(X) d V
\end{aligned}
$$

where, $W_{i j}\left(X^{\prime}, x\right), P_{i j}\left(X^{\prime}, x\right)$ and $\dot{\succ}_{\mathrm{ij}}\left(X^{\prime}, X\right)$ are called fundamental solutions representing a displacement, a traction and strain in the $j$ direction at point $X$ due to a unit point force in the $i$ direction at point $X^{\prime}$. These fundamental solutions can be found in Karam (1998). $\dot{q}(X), \dot{M}_{\alpha \beta}^{p l}(X)$ are the load rates and the plastic rate terms due to the loading.respectively.

Equation (1) is valid for any source points within domain $\left(X^{a} V\right)$, in order to find solutions on the boundary points, it is necessary to consider the limiting process as $X^{\prime \prime} ! x^{\prime a} S$. After limiting process, boundary displacement integral equations can be expressed as

$$
\begin{aligned}
C_{i j}\left(x^{\prime}\right)\left(\dot{w}_{i}\left(x^{\prime}\right)=\right. & \int_{S} W_{i j}\left(x^{\prime}, x\right) \dot{p}_{j}(x) d S-\int_{S} P_{i j}\left(x^{\prime}, x\right) \dot{w}_{j}(x) d S+ \\
& \int_{V} W_{i 3}\left(x^{\prime}, X\right) \dot{q}_{3}(X) d V+ \\
& \int_{V} \chi_{\alpha \beta i}\left(x^{\prime}, X\right) \dot{M}_{\alpha \beta}^{p l}(X) d V
\end{aligned}
$$

where, $C_{i j}\left(x^{\prime}\right)$ is free term that is $C_{i j}\left(x^{\prime}\right)=\ddot{a}_{i j}\left(x^{\prime}\right)$ $+\dot{a}_{i j}\left(x^{\prime}\right)$, for smooth boundary the free term is 0.5 .

The Somigliana's identity for stresses can be expressed respectively as

$$
\begin{aligned}
\dot{M}_{\alpha \beta}\left(X^{\prime}\right)= & \int_{S} W_{\alpha \beta k}\left(X^{\prime}, x\right) \dot{p}_{k}(x) d S-\int_{S} P_{\alpha \beta k}\left(X^{\prime}, x\right) \dot{w}_{k}(x) d S \\
& +\int_{V} W_{\alpha \beta k}\left(X^{\prime}, X\right) \dot{q}_{3}(X) d V \\
& +\int_{V} \chi_{\alpha \beta \gamma \theta}\left(X^{\prime}, X\right) \dot{M}_{\gamma \theta}^{p l}(X) d V \\
& -\frac{1}{8}\left[2(1+v) M_{\alpha \beta}(1-3 v) \dot{M}_{\theta \theta}^{p l} \delta_{\alpha \beta}\right]
\end{aligned}
$$




$$
\begin{aligned}
Q_{\beta}\left(X^{\prime}\right)= & \int_{S} W_{\alpha \beta k}\left(X^{\prime}, x\right) p_{k}(x) d S-\int_{S} P_{\alpha \beta k}\left(X^{\prime}, x\right) w_{k}(x) d S \\
& +\int_{V} W_{3 \beta k}\left(X^{\prime}, V\right) q_{3}(X) d V \\
& +\int_{V} \chi_{3 \beta \gamma \theta}\left(X^{\prime}, X\right) \dot{M} \dot{\gamma}_{\gamma \theta}^{p l}(X) d V
\end{aligned}
$$

where, $M_{\dot{a} \hat{a}}$ and $\mathrm{Q}_{\mathrm{a}}$ are moment and shear stresses respectively. $W_{i \hat{a} \hat{k}}\left(X^{\prime}, x\right), P_{\dot{a} \hat{a} k}\left(X^{\prime}, x\right)$ and $\dot{\bar{a}}_{\bar{a} \hat{e} \mathrm{e} k}\left(X^{\prime}, X\right)$ are called fundamental solutions and can be found in Karam (1998).

\section{Dicretization and System of Equation}

In order to solve equation (1), (2), (3) and (4), a numerical method is implemented. The boundary $S$ is discretized using quadratic isoparametric elements. The domain $V$ is divided into number of cells of 9 nodes quadrilateral cell.

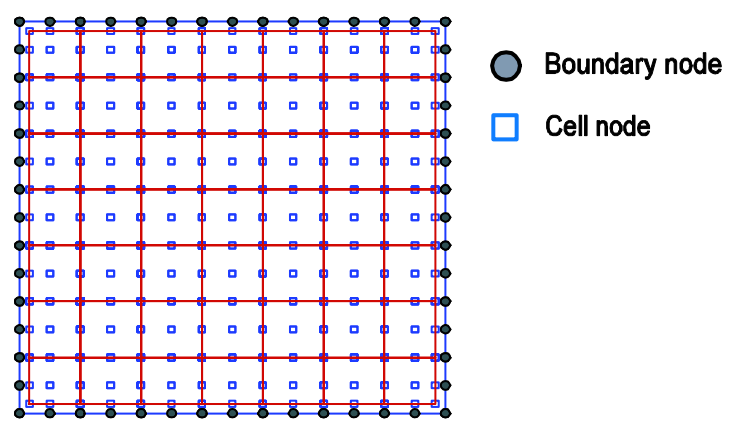

Figure 1. Discretization

In this formulation, boundary parameter $x_{j}$, the unknown boundary values of displacements $w_{j}$ and tractions $p_{j}$ are approximated using interpolation function, in following manner:

$$
\begin{aligned}
& x_{j}=\sum_{\alpha=1}^{3} N_{\alpha}(\xi) x_{j}^{\alpha} \\
& \dot{w}_{j}=\sum_{\alpha=1}^{3} N_{\alpha}(\xi) \dot{w}_{j}^{\alpha} \\
& \dot{p}_{j}=\sum_{\alpha=1}^{3} N_{\alpha}(\xi) \dot{p}_{j}^{\alpha}
\end{aligned}
$$

The shape functions $N_{\dot{a}}$ are defined as

$$
\begin{aligned}
& N_{1}=\frac{1}{2} \xi(\xi-1) \\
& N_{2}=(1-\xi)(1+\xi) \\
& N_{3}=\frac{1}{2} \xi(\xi+1)
\end{aligned}
$$

Substituting equation (5) and equation (6) into equation (2), one gets (the integrations on the boundary $S$ ):

$$
\begin{aligned}
& \int_{S} P_{i j}\left(x^{\prime}, x\right) w_{j}(x) d S=\sum_{n=1}^{N e} \sum_{\alpha=1}^{3} w_{j}^{\alpha} \int_{-1}^{1} P_{i j}\left(x^{\prime}, x(\xi)\right) N_{\alpha}(\xi) J^{n}(\xi) d \xi \\
& \int_{S} W_{i j}\left(x^{\prime}, x\right) p_{j}(x) d S=\sum_{n=1}^{N e} \sum_{\alpha=1}^{3} p_{j}^{\alpha} \int_{-1}^{1} W_{i j}\left(x^{\prime}, x(\xi)\right) N_{\alpha}(\xi) J^{n}(\xi) d \xi
\end{aligned}
$$

where, $\mathrm{Ne}$ is the number of elements on the boundaries $S$ and $J^{n}$ is the Jacobian transformations.

After discretization process the integration on the domain $V$ can be stated as:

$$
\begin{aligned}
& \int_{V} W_{i 3}\left(x^{\prime}, X\right) q_{3}(X) d V=\sum_{n=1}^{N c} \sum_{\alpha=1}^{3} q_{3} \int_{-1}^{1} W_{i 3}\left(x^{\prime}, X(\xi, \eta)\right) \\
& N_{\alpha}(\xi, \eta) J^{n}(\xi, \eta) d \xi d \eta \\
& \int_{V} \chi_{\alpha \beta i}\left(x^{\prime}, X\right) \dot{M}_{\alpha \beta}^{p l}(X) d V=\sum_{n=1}^{N c} \sum_{\alpha=1}^{9}\left(\dot{M}_{\alpha \beta}^{p l}\right)_{\alpha}(X) \times \\
& \int_{-1}^{1} \chi_{\alpha \beta i}\left(x^{\prime}, X(\xi, \eta)\right) N_{\alpha}(\xi, \eta) J^{n}(\xi, \eta) d \xi d \eta
\end{aligned}
$$

After discretization and point collocation passes through all the collocation node on the boundary as well as in the domain, the equations (2) can be written in the matrix form as

$$
[H]\{\dot{w}\}=[G]\{\dot{p}\}+\{\dot{b}\}+[T]\left\{\dot{M}^{p l}\right\}
$$

where $[\mathrm{H}]$ and $[\mathrm{G}]$ are the well-known boundary element influence matrices, $[T]$ is the influence matrix due to plasticity. $\{. w\},\{. p\}$, are the displacement and the traction rate vectors on the boundary . $\{b\}$ is the load rate vectors on the domain and $\left\{M^{p l}\right\}$ is the nonlinear term due to plasticity.

After imposing boundary condition, equations (9) can be written as

$$
[A]\{\dot{x}\}=\{f\}+[T]\left\{\dot{M}^{p l}\right\}
$$

where, $[A]$ is the system matrix, $\{x\}$ is the unknown vector and $\{f\}$ is the vector of prescribed boundary values.

Analogously, the stress integral equations of equations (3) and (4) can be presented in matrix form as
$\left[\begin{array}{c}\dot{M} \\ \dot{Q}\end{array}\right]=[G]\{\dot{p}\}-[H]\{\dot{w}\}+\{\dot{b}\}+[T]\left\{\dot{M}^{p l}\right\}$ 


\section{Solution Algorithm}

The total incremental method solves the nonlinear system of equations of equation (10) based on the incremental load to be applied on the structure. It has an algorithm as:

1. Solve the equation (10), assume that the nonlinear term $M^{p l}=0$ for the first load increment. It means that the linear system equations are solved. For the $(k+1)$ th load increment it is assumed that $\left(M^{p l}\right)_{(k+l) \text { th }}=\left(M^{p l}\right)_{k \text { th }}$

2. Solve equation (11) based on the boundary values obtained from number 1 . The same case as number 1 is implemented for the nonlinear term.

3. Evaluate of the plastic zone based on the stress obtained from the number 2 . In this stage the von Mises criterion is used.

4. If the plasticity has taken place then, obtain the nonlinear term otherwise go to the number 5. The clear explanation of the determination of the plastics term can be found in the work by Karam (1998).

5. If the load is less than the final load then go to number 1 and repeat until the load is equal final load

\section{Numerical Example}

In order to show how the total incremental method has a good agreement to the incremental and iterative method in solving the nonlinear system of equation due to plasticity, some examples are presented.

\section{Simply supported circular plate}

A simply supported circular plate of radius $\mathrm{a}=10.0 \mathrm{inc}$ and thickness $\mathrm{h}=1 \mathrm{inc}$, is subjected to a uniformly distributed load q (see Fig.2). It is assumed that the plate is elastic perfectly plastic material with $E=10^{4} \mathrm{ksi}, o_{y}=16 \mathrm{ksi}$ and $i^{\prime}=0.24$. Due to symetry conditions, only a quarter of the plate is discretized. Two different BEM meshes are used. The first mesh A has 12 boundary elements and the second mesh B has 18 boundary elements. In order to simulate the plasticity effects, the domain is also meshed into 12 cells of 9-nodes quadrilateral for the first mesh A and 24 cells for the second mesh $B$. The load increment of $\ddot{A} q=0.00268$ and $\ddot{A} q=0.00134$ are implemented. These load increments are the same as 100 and 200 steps respectively to reach the final load.
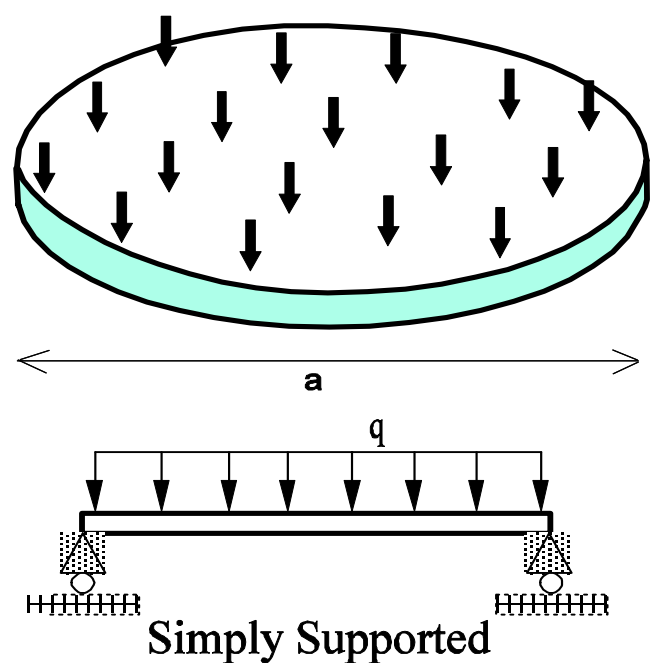

Figure 2. simply supported circular plate

The results are presented in Figures 3 and 4. The abscise and ordinate of the graphs are presented in non-dimensional parameter as

$$
Q=\frac{\Delta q a^{2}}{M_{0}} \text { and } W=\frac{w D}{M_{0} a^{2}}
$$

where $M_{0}=o_{y} h^{2} / 4, w$ is the deflection on center plate and $D=E h^{3} / 12\left(1-i^{2}\right)$

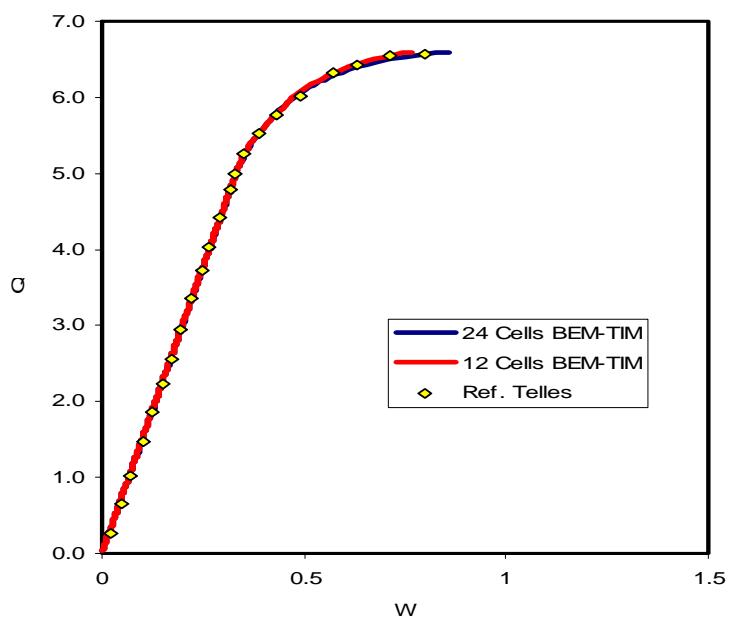

Figure 3. Circular plate. Load-deflection curve $\ddot{A} q=0.00134$. 


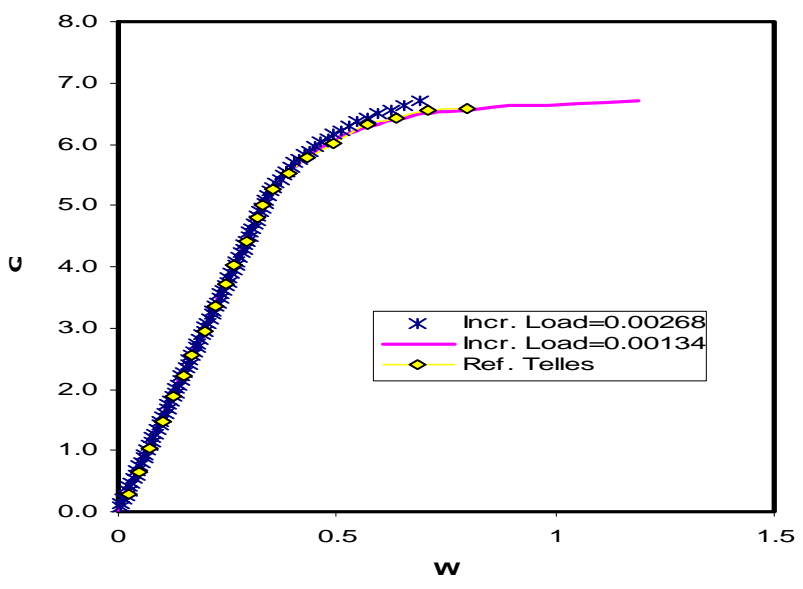

Figure 4. Load-deflection curve for $\ddot{A} q=0.00268$ and $\ddot{A} q=0.00134$ of mesh $B$

\section{Simply supported square plate}

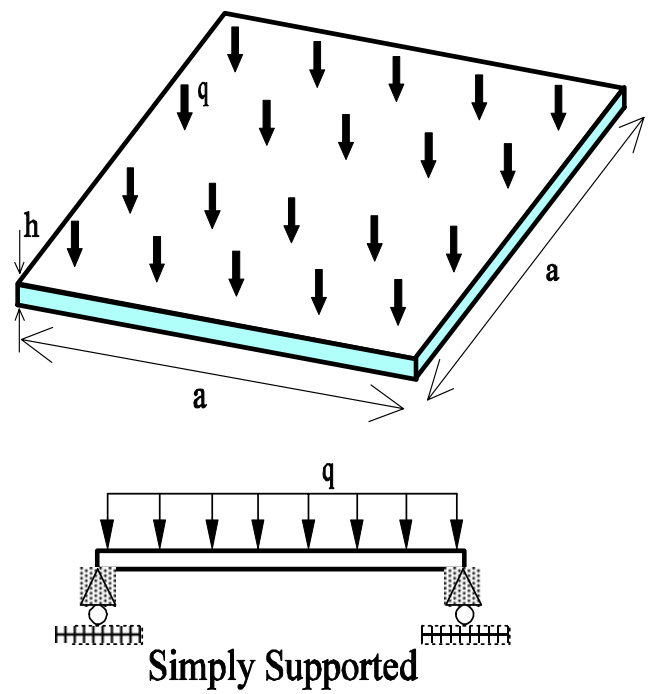

Figure 5. simply supported square plate

A simply supported square plate having side $\mathrm{a}=1$ and thickness $\mathrm{h}=0.01$, is subjected to a uniformly distributed load q. As in the circular plate, it is assumed that the plate undergoes elastic perfectly plastic material with $E=10.92$ $\mathrm{ksi}, o_{y}=1600 \mathrm{ksi}$ and $i=0.3$. Also only a quarter of the plate is discretized due to simetry conditions. Two different BEM meshes are used. The first mesh A has 16 boundary elements and the second mesh B has 20 boundary elements. In order to simulate the plasticity effects, the domain is also meshed into 16 cells of 9-nodes quadrilateral for the first mesh A and 25 cells for the second mesh $\mathrm{B}$. The load increment of $\ddot{A} q=0.005$ is implemented and the same as 200 steps to reach the final load.

The results are presented in figures 4 and the non-dimensional parameters are

$$
\text { and } W=\frac{10^{2} w D}{M_{0} a^{2}}
$$

where $M_{0}=\sigma_{y} h^{2} / 4, w$ is the deflection on center plate and $D=E h^{3} / 12\left(1-v^{2}\right)$

The curves of the both meshes A and B are in a good agreement with the work done by Telles (1998).

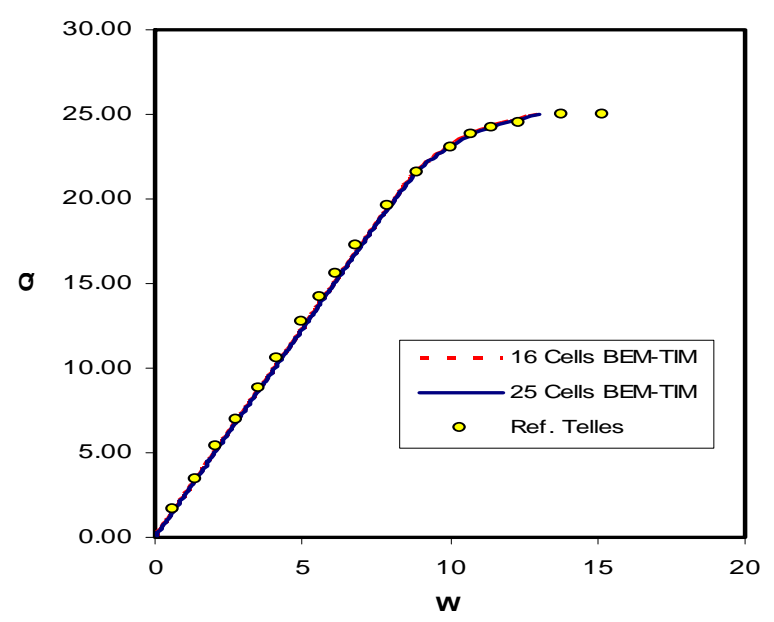

Figure 6. Square plate. Load-deflection curve Ä $q=0.005$.

\section{CONCLUSION}

The application of BEM to material nonlinearity for shear deformable plate bending analysis was presented and the total incremental method was implemented to solve the nonlinear system of equation, from the results obtained it can be concluded that:

1. The total incremental method was shown to be an efficient approach for this problem as 
repeated solution of system of equations is not required and the nonlinear terms are updated by back substitution.

2. The size of load increment shows big influence on the results. The smaller the size the better results can be obtained, however 200 steps to reach the final load is a reasonable size to get a good results.

\section{REFERENCES}

Aliabadi, M.H., Plate Bending Analysis with Boundary Element, Computational MechanicsPublications, Southampton (1998).

Dirgantara, T. and Aliabadi, M.H., Boundary element formulation for geometrically non-linear analysis of shear deformable shells, Submitted for publication (2006)

Kamiya, N. and Sawaki, Y., An integral equation approach to finite deflection of elastic plates, Int. J. Non-Linear Mech., 17(3), 187-194 (1982) 19

Karam, V.J. and Telles, J.C.F., On boundary elements for Reissner's plate theory,Engineering Analysis, 5, 21-27 (1988)

Karam, V.J. and Telles, J.C.F., Nonlinear material analysis of Reissner's plates, Plate Bending Analysis with Boundary Element, 127-163, Computational Mechanics Publications, Southampton (1998).

Kirchhoff, G., Uber das gleichgewicht und die bewegung einer elastischen scheibe, J.Rein Angew Math., 40, 51-88 (1850)

Lei, X.Y., Huang, M.K. andWang, X.X., Geometrically nonlinear analysis of a Reissner's type plate by boundary element method, Comput. Struct., 37(6), 911-916 (1990)

Naghdi, P.M., On the theory of thin elastic shells, Quarterly of Applied Mathematics, 14, 369-380, 1956.

Purbolaksono, J. and Aliabadi, M.H., Large deformation of shear deformable plate by boundary element method, J. of Engineering Mathe matics, (2005)

Reissner, E., On a variational theorem in elasticity, Journal of Mathematics and Physics, 29, 90-95, (1950).

Ribeiro, GO. and Venturini,W.S., Elastoplastic analysis of Reissner's plate using the boundary element method, Plate Bending Analysis with Boundary Element, 101-125, Computational Mechanics Publications, Southampton (1998).

Supriyono, Aliabadi, M.H., Boundary Element Method for Shear Deformable Plates with Combined Geometric and Material Nonlinearities, Engineering Analysis with Boundary Elements, 30, 31-42 (2006). 
Tanaka, M., Large deflection analysis of thin elastic plates, Developments in Boundary Element Methods, Elsevier Applied Science Publishers, 13, 115-136 (1984)

Wen, P.H., Aliabadi, M.H., Young, A., Large deflection analysis of Reissner plate byboundary element method, Computer \& Structure, (2004) 